\title{
Liquid-liquid phase separation in concentrated solutions of non-crystallizable polymers by spinodal decomposition
}

\author{
By C.A.Smolders, J.J.van Aartsen, and A. Steenbergen,
}

With 11 figures

\section{Introduction}

In the past, investigations of phase separation in concentrated polymer solutions centered first of all on the crystallization kinetics (1) and morphology (2) of polymer erystals growing from solutions and, secondly, on the equilibrium thermodynamic properties of liquid-liquid phase separation phenomena (3).

Papkov and Yefimova (4) have recentily given a classification of different types of phase separation for polymer solutions, essentially the two types mentioned above, in which they also pay some attention to the difference in final state of separation (i. e. "gel" or two liquid layers) and to the mechanism of separation itself. For liquidliquid phase separation of solutions of noncrystallizable polymers the difference in final state is ascribed to a combined effect of the thermodynamies of the system (the width of the miscibility gap in the temperature-composition diagram) and the flow properties of the concentrated polymer phase. In their own words: "For amorphous or barely crystallizing polymers, gel formation is only to be expected if the flow temperature of the polymer is high enough and the low molecular weight component does not reduce this point too much". The authors' view on the mechanism of separation can be summarized as follows: the gel is formed by separation and growth of microsections of the dilute polymer phase, leaving the concentrated polymer phase as a gradually stiffening matrix, until its final equilibrium concentration is reached. This is the well-known mechanism of nucleation and growth of one of the equilibrium phases.

Recently, Cahn and others $(5,6)$ have described a second type of mechanism of phase separation: the spinodal decomposition of a homogeneous solution. This mechanism may lead to typical network structures in isotropic media, as has been shown for inorganic glasses (7).
(Received June 15, 1970)

We will describe here under what conditions spinodal decomposition can be expected for polymer solutions. A number of observations on phase separation in concentrated solutions of poly(2,6-dimethyl-1,4phenylene ether), $\left(\mathrm{PPO}^{\circledR}\right), T_{g}=210^{\circ} \mathrm{C}$, and of an ethylene-vinyl acetate copolymer (Elvax-40), $T_{g}=0^{\circ} \mathrm{C}$, will be reported, which will make it clear that spinodal decomposition can be realized in polymer solutions.

\section{Thermodynamic considerations}

Fig. $1 \mathrm{a}$ and $\mathrm{b}$, gives a schematic representation of the thermodynamics of liquid-
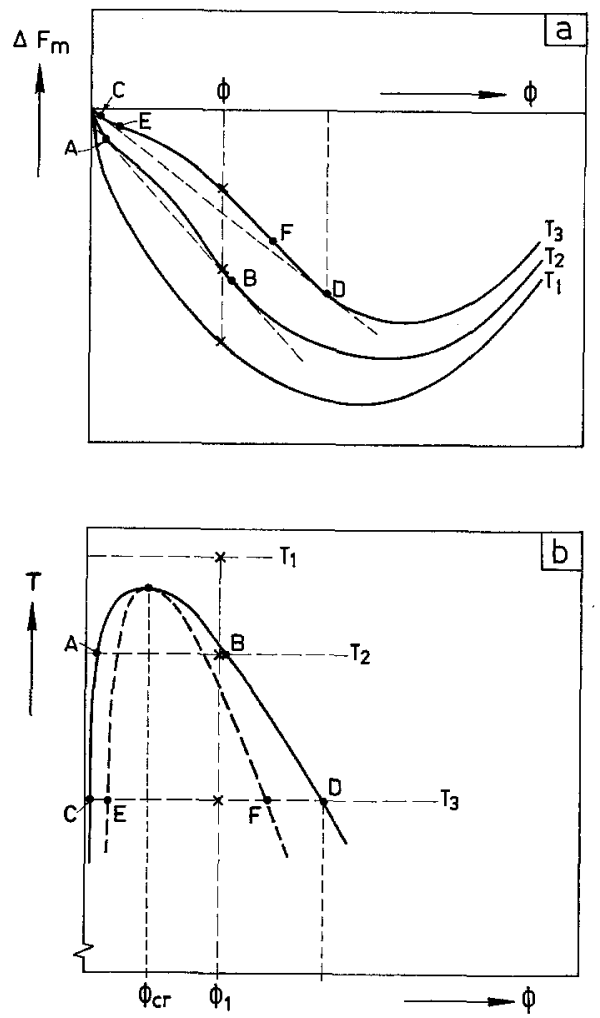

Fig. 1. Diagrams of the free energy of mixing $\left(\Delta F_{m}\right)$ and temperature $(T)$ versus composition $(\Phi)$ of a two com. ponent system polymer-solvent with a miscibility-gap 
liquid phase separation in polymer solutions (two components). Lowering the temperature of a homogeneous solution of composition $\Phi_{1}$ at $T_{1}$ to below the temperature $T_{p}$ (on the binodial curve) makes phase separation energetically favourable. When equilibrium has been reached the separated phases will have attained the compositions $\Phi_{A}$ and $\Phi_{B}$ at $T_{2}$, $\Phi_{C}$ and $\Phi_{D}$ at $T_{3}$, these compositions being given by the common tangents of the $\Delta F_{m^{-}} \Phi$ curves at these temperatures. The binodial curve in the temperature-composition diagram thus collects all the equilibrium compositions.

Another set of characteristic points on the $\Delta F_{m}-\Phi$ curves are the inflection or spinodal points, e.g. points $E$ and $F$ at $T_{3}$. These points mark the composition region within which the system, after rapid cooling to $T_{3}$, is unstable as a homogeneous system, whereas in the composition regions $C E$ and $F D$ the system is metastable at $T_{3}$. The inflection points in the $\Delta F_{m^{-}} \Phi$ diagram at different temperatures collect to give the spinodal curve of the temperature-composition diagram (dotted line in fig. $1 \mathrm{~b}$ ).

\section{Two mechanisms of liquid-liquid phase separa- tion}

Now apparently two different mechanisms of phase separation are possible:

\section{Nucleation and growth}

In the regions between the binodial and spinodal curves in the $T-\Phi$ diagram the solution is stable to ordinary small amplitude fluctuations in concentration, but unstable to concentration fluctuations (which may be called nuclei) having compositions close to the other extreme of the miscibility gap. Such a nucleus will spontaneously grow in size as soon as its radius has surpassed a critical value, following the familiar pattern known from liquid separation from the gas phase and from crystallization phenomena. Eventually the solution will become a dispersion of droplets of one of the equilibrium phases in the other one.

For concentrated polymer solutions with $\Phi>\Phi_{\text {cr }}$ (while $\Phi_{\text {er }} \leqslant 10 \%$ by weight), one expects nuclei of the practically pure solvent phase to be formed. Depending on the flow properties of the polymer phase matrix, which increases in concentration, a more or less rigid mass with solvent-filled holes or, at the other extreme, two liquid layers, one practically pure solvent, the other rich in polymer, would be formed. This is in accordance with the picture usually given $(3,4)$ for phase separation in solutions of amorphous polymers.

Is should be realized that, especially at polymer concentrations higher than a few percent, undercooling below the binodial curve can easily occur before effective nucleation is possible. This may be a reason why so-called cloud point curves lie below the binodial branches for high polymer concentrations ${ }^{1}$ ).

\section{Spinodal decomposition}

In the composition region within the spinodal curve in the $T$ - $\Phi$ diagram the solution will not be stable to fluctuations in concentration, of however small an amplitude. The theory of spinodal decomposition as given by Cahn (5) predicts that small fluctuations in concentration of wavelengths above a certain critical value, $\Lambda_{c}$, will spontaneously increase in amplitude. This means that the difference in concentration in adjacent regions in the solution, at fixed distances apart, increases in a continuous way. Cahn also showed that, although all concentration fluctuations of $A>\Lambda_{c}$ do increase in amplitude with time, the rate of increase depends on the fluctuation wavelength. Fluctuations of a wavelength $D_{m}=\Lambda_{c} \sqrt{2}$ will grow fastest.

The theory describes the earliest stages of the process only. If the spinodal decomposition pattern is conserved in later stages, which can be expected for strongly increasing viscosity of the regions increasing in polymer concentration, a fairly uniform structure will result, with a length parameter $D_{m}$ occurring most frequently as the distance between polymer-rich regions. The morphological structure to be expected from the spinodal mechanism is a more or less rigid mass consisting of interconnected regions of the concentrated polymer phase, embedded in the dilute polymer phase (i. e. practically pure solvent.) Cahn suggests that the relative volume fraction of the minor phase present

1) We would like to point out here that as a model for concentrated polymer solutions such a system should preferably be considered as an assembly of polymer segments and solvent molecules with a practically homogeneous distribution of segments over the solution. Concentration fluctuations can then be described as fluctuations in segment density or as fluctuations in the concentration of solvent molecules. Hence the influence of the properties of the individual polymer molecules (e. g. their m. w. distribution) on nucleation phenomena and on the cloud point curve - as is experienced at low polymer concentrations - becomes unimportant at high polymer concentrations. 
should at least be $15 \%$ in order to maintain interconnectivity of this phase. For polymer solutions with a typical asymmetrical binodial curve this means that this interconnected structure can already be expected to be realizable above a few percent of polymer, by weight or volume. As an example: taking the binodial compositions at about $0 \%$ and $30 \%$ volume of polymer respectively, the separated mixture contains more than $15 \%$ by volume of the polymer phase if the total polymer content of the mixture is above $4.5 \%$ by volume. At a lower polymer content spinodal decomposition would give separated polymerrich regions, a distance $D_{m}$ apart.

If the polymer-rich phase has sufficient mobility at all concentrations, the primary regions may form larger aggregates and eventually the splitting up into two bulk liquid phases becomes possible. Also the case of a contracting gel phase (8) covered by a layer of the "solvent" phase of changing composition can easily be explained on the basis of the gel structure formed in an early stage and at relatively low total polymer concentration by the spinodal decomposition mechanism.

\section{Estimation of the structure parameter $D_{m}$ from theory}

Combining Debye's theory on the thermodynamies and critical opalescence of polymer solutions, in which random concentration fluctuations occur, with the theory of spinodal decomposition as given by $C a h n$, we have derived (10) the following expression for $D_{m}$

$$
D_{m}=2 \pi l /\left[3\left(1-T / T_{s}\right)\right]^{1 / 2},
$$

where $T$ is the temperature at which a solution of concentration $\Phi$ is quenched, $T_{s}$ is the spinodal temperature at that concentration, and $l$ is a "range of molecular interaction" as defined by Debye. Debye further estimates that $l$ should be equal to the radius of gyration of the polymer. Thus $l$, and therefore $D_{m}$, depends on the solvent quality, on the total polymer concentration, etc. For some polymers values of $l$ ranging from 50-100 $\AA$ have been reported. For a solution of PPO $\left(\bar{M}_{v \text { (chloroform) }}=2.1 \times 10^{5}\right)$ in caprolactam $(\mathrm{CL})$ at a 15 weight percent concentration $l$ should be of the order of $200 \AA$. Taking $T_{s} \approx 400^{\circ} \mathrm{K}$, we calculate values of $D_{m}=1.45 \mu$ at $1^{\circ}$ undercooling, $D_{m}=0.65 \mu$ at $5^{\circ}$ undercooling and $D_{m}=0.26 \mu$ at $30^{\circ}$ undercooling below $T_{s}$. These structures should become visible under a normal light microscope.

\section{Survey of observations}

We have made a number of observations (among which light scattering measurements, dilatometry, normal and electron microscopy) on concentrated polymer solutions, upon quenching these solutions to sufficiently low temperatures for phase separation to occur. The results of these observations can be explained by a spinodal decomposition mechanism at early stages of the phase separation process, followed by other, slow phenomena at high enough quenching temperatures.

Since not enough thermodynamic data were available to compute the spinodal temperatures at different compositions, we had to rely on other methods for estimating these properties (see below).

We want to stress again that concentrated solutions may easily be undercooled below the binodial curve without phase separation of the nucleation and growth type taking place. On the other hand, phase separation always occurs below the spinodal temperature, since then the homogeneous system is unstable. This favours the spinodal decomposition mechanism to be found if the quenching technique is used: rapid exchange of heat is promoted by the use of small, thin samples of the solution and a quenching medium of large heat capacity.

\section{Short-time versus long-time effects}

Light scattering measurements (9) at different angles, carried out on a solution of PPO (15\% by weight) in caprolactam quenched at several temperatures between $128^{\circ} \mathrm{C}$ and $55^{\circ} \mathrm{C}$, have given an intensity vs time pattern as sketched in fig. 2.

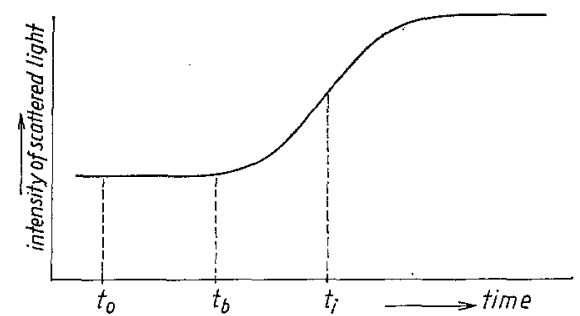

Fig. 2. Schematic representation of light scattering intensity as a function of time, after quenching a PPO/ Caprolactam solution

At time $t_{0}$ the thin sample was quickly brought from an initial temperature of $190^{\circ} \mathrm{C}$ to the desired temperature, the whole temperature change not taking more than a few seconds. After an induction period $t_{b}-t_{0}$ the 
light scattering intensity was seen to increase to a new, practically constant, higher level. An important point is that the change in intensity is completed within a few minutes at the most, the higher the temperature, the longer the period.

Two further effects of phase separation, which take much longer time, however, have been observed after quenching at temperatures between $100^{\circ} \mathrm{C}$ and $130^{\circ} \mathrm{C}$.

In the first place, dilatometric measurements show that the large change in volume occurring upon quenching a 1 ce sample from $180^{\circ} \mathrm{C}$ to lower temperatures (this first step taking about $10 \mathrm{~min}$ ) is followed by a second volume effect on a time scale ranging from hours at high quenching temperatures to minutes at lower temperatures, see fig. 3. At quenching temperatures of $90^{\circ} \mathrm{C}$ and lower these secondary effects probably fall within the first period of $10 \mathrm{~min}$ necessary to reach the desired temperature.

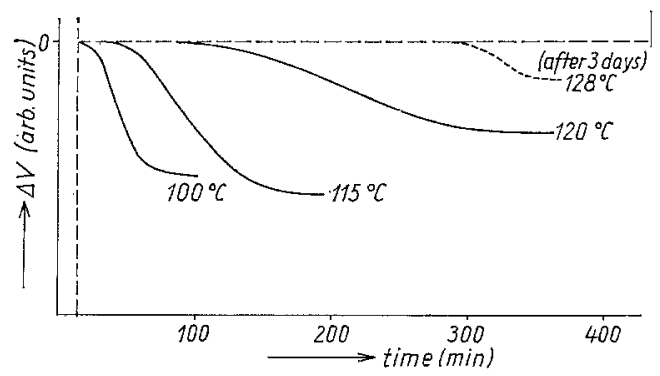

Fig. 3. Volume change $(\Delta V)$ per unit weight of a $15 \%$ PPO solution in Caprolactam quenched at different temperatures. The volume change in the first $10 \mathrm{~min}$ after quenching has been excluded

Secondly, typical after-effects were found by microscopic observation of small samples taken from 5 cc solutions of PPO in caprolac- tam, which had been quenched at temperatures between $110^{\circ} \mathrm{C}$ and $130^{\circ} \mathrm{C}$. Droplets of the polymer phase (identified by centrifugation) at these high temperatures ${ }^{2}$ ) were seen to increase in size with time. The number of droplets and their growth rate are low at high temperatures. Both increase as the quenching temperatures have been lower, until at $110^{\circ} \mathrm{C}$ and less the whole phenomenon falls within the quenching period, which too was $10-15 \mathrm{~min}$. The initial growth rate of the droplets depends on temperature and concentration (fig. 4, a and b); all data on initial growth rates are collected in fig. $4 \mathrm{c}$.

At first sight one would interpret these effects as phase separation by nucleation and growth. This, however, does not agree with the quantitative interpretation of the light scattering results (9) at early stages of the decomposition. Furthermore, for the polymer phase to be nucleated the critical concentration would have to be higher than $15 \%$ of polymer, which is unlikely. We believe that these long time effects can be ascribed to the process of reaching final equilibrium compositions in the two liquid phases; they are not typical of the onset of separation as measured by light scattering and studied further below.

\section{Observations under the light microscope}

On quenching thin samples of solutions of $5-20 \%$ PPO in caprolactam to temperatures between $50{ }^{\circ} \mathrm{C}$ and $120^{\circ} \mathrm{C}$, using a light microscope with a heating stage, one obtains patterns of phase separation as shown in

$\left.{ }^{2}\right)$ Centrifugation at a few thousand times $g$ does not bring about any separation into two layers if the PPO concentration is high $(>10 \%)$ and the quenching temperature is below about $100^{\circ} \mathrm{C}$.



$\underline{A}$

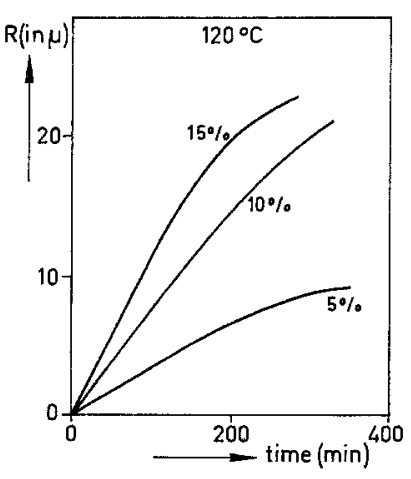

B

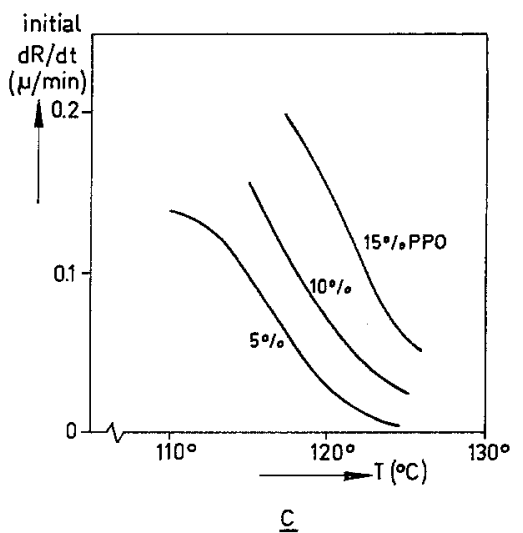

Fig.4c. Initial growth rate data as a function of temperature and concentration
Fig. $4 a$, b. The change of droplet radius $(R)$ with time at different concentrations and temperatures 
fig. 5. These patterns appear within times varying from a few seconds at low temperature to a few minutes at high temperature. They change in intensity (not in geometrical arrangement) within a few minutes at the most (see fig. 6), just as in light scattering experiments.

The dimension parameter estimated from micrographs of a $15 \%$ PPO solution gives patterns as given in figs. 5 and 6 originate from spinodal decomposition of the solutions involved.

There remain two interesting points to be discussed : first, an estimation of the spinodal temperatures in the system PPO/CL and, secondly, the applicability to other systems, which will be briefly discussed for the system Elvax-40/CL.

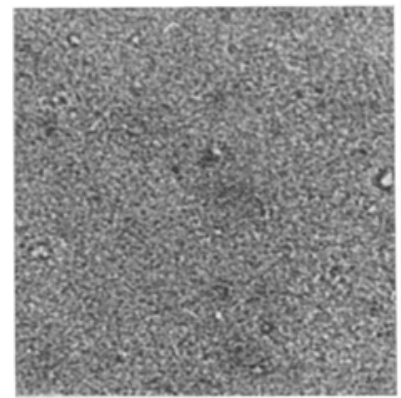

$50^{\circ} \mathrm{C}$

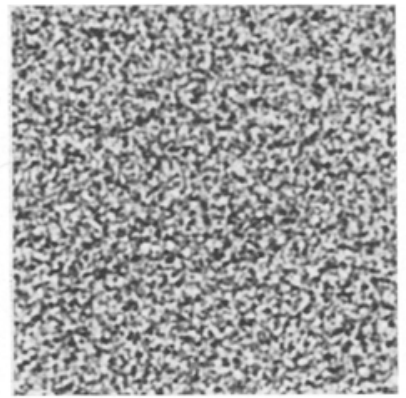

$100^{\circ} \mathrm{C}$

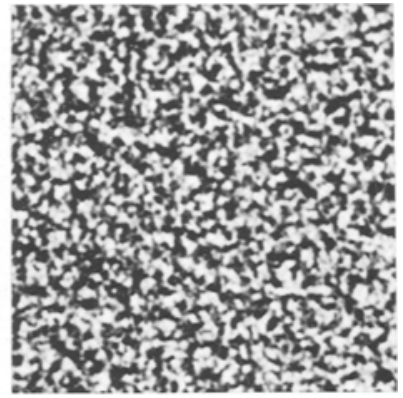

$120^{\circ} \mathrm{C}$

Fig. 5. Micrographs of final structures of a $20 \%$ PPO/CL solution quenched at different temperatures

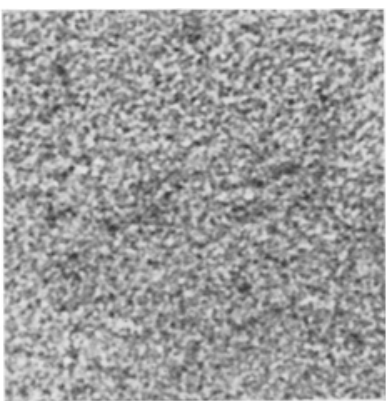

$20 \mathrm{sec}$

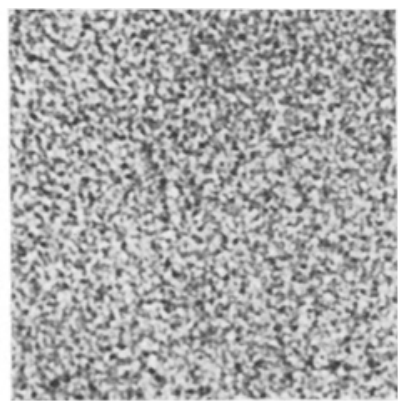

$40 \mathrm{sec}$

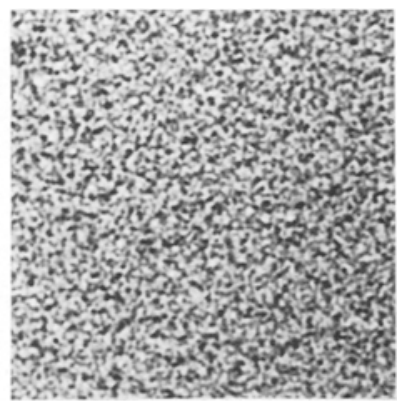

$60 \mathrm{sec}$

Fig. 6. Time study of phase separation in a $20 \%$ PPO/CL solution quenched at $100^{\circ} \mathrm{C}$

values from about $0.3 \mu$ at $50{ }^{\circ} \mathrm{C}$ to about $2 \mu$ at $120^{\circ} \mathrm{C}$ quenching temperature. These dimensions agree very well with the structure parameter obtained from theory (eq. 1). Also the quantitative evaluation of the light scattering data (9), applying the kinetic model obtained from the theory of spinodal decomposition, gives values for $D_{m}$ which are of the same order of magnitude: $0.7 \mu$ at $55^{\circ} \mathrm{C}$ to $1.0 \mu$ at $128{ }^{\circ} \mathrm{C}$. Finally, electron microscopy of quenched solution samples which were frozen at low temperatures $\left(-180^{\circ} \mathrm{C}\right)$ after a certain quenching time at $90{ }^{\circ} \mathrm{C}$ gives patterns of decomposition with a structure parameter of about $1 \mu$. We therefore believe that the typical decomposition

\section{Spinodal temperatures in the system PPO/CL}

On microscopic examination of the phase separation phenomena it was observed that the time which elapses before the spinodal pattern appears is short (seconds) at low quenching temperatures. This "induction time" suddenly increases to high values at temperatures not far from $130^{\circ} \mathrm{C}$ for solutions containing $10-25 \%$ PPO (see fig. 7). Now, above the spinodal temperature appearance of the familiar separation pattern cannot be expected; on the other hand, for solutions of PPO in CL nucleation phenomena have never been observed within reasonable time limits. The temperatures at which it 


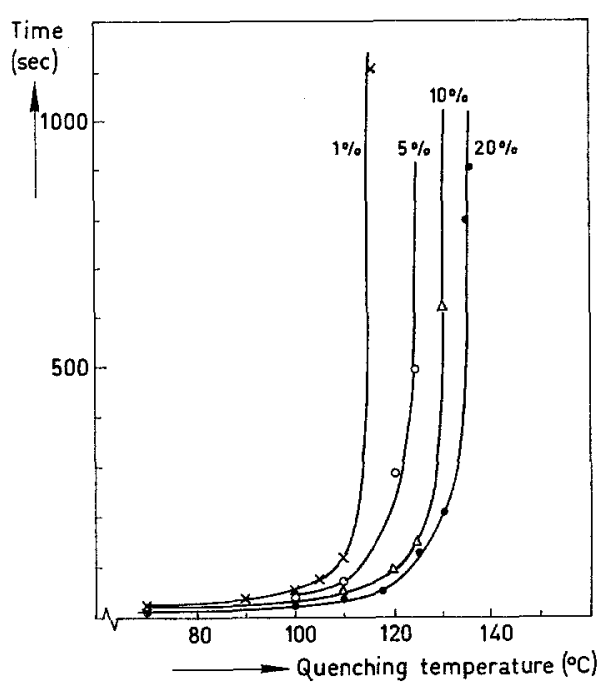

Fig. 7. Time of appearance of turbidity in thin samples of solutions of $\mathrm{PPO} / \mathrm{CL}$ quenched at different temperatures

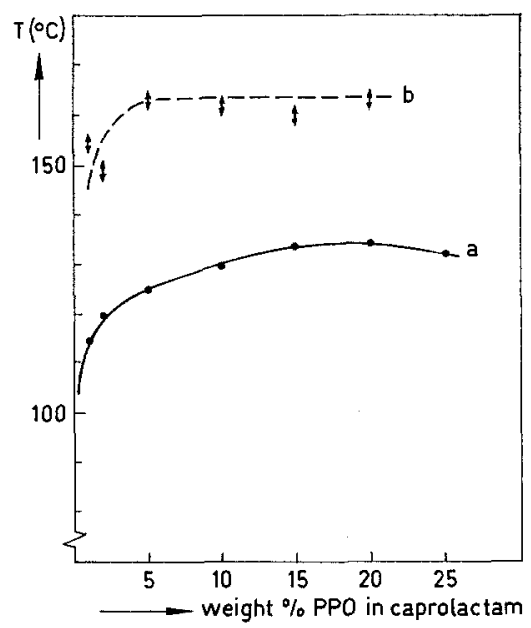

Fig. 8. Curve a: spinodal temperatures based on $10 \mathrm{~min}$ turbidity points from fig. 7; curve $b$ : disappearance of turbidity took more than 10 min for any turbidity to occur have therefore been taken as an estimate of the spinodal temperatures for several compositions in the system PPO/CL. The spinodal curve thus obtained is given in fig. 8 .

This figure also shows the temperatures at which the phase separation disappears on heating. The high dissolution temperatures found here might either indicate points belonging to the binodial branches of the solutions investigated, or might stem from a possible change of the polymer molecules to the glassy state after phase separation. In the case of this obviously bad solvent for PPO, the polymer could perhaps dissolve from the glassy state at relatively high temperatures.

\section{Phase separation in other systems: Elvax-40/CL}

The system Elvax-40/CL gives phase separation with two liquid layers in the final state. Rapid coarsening of the primary structure is already seen upon quenching to temperatures between $70^{\circ} \mathrm{C}$ and $120^{\circ} \mathrm{C}$. The temperatures where turbidity sets in and where it disappears are given in fig. 9 .

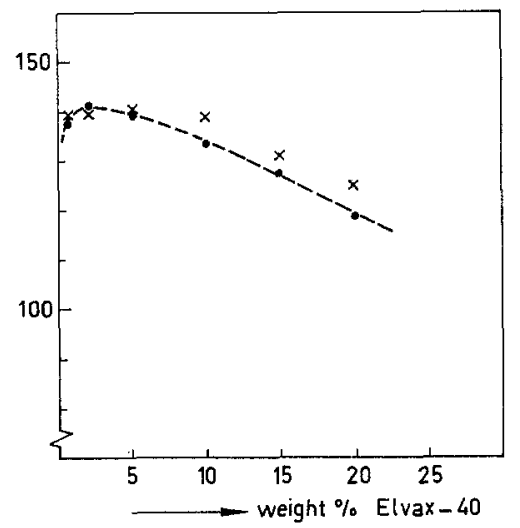

Fig. 9. Turbidity points (dots) for thin samples of Elvax-40/CL. Crosses indicate temperatures at which turbidity disappears

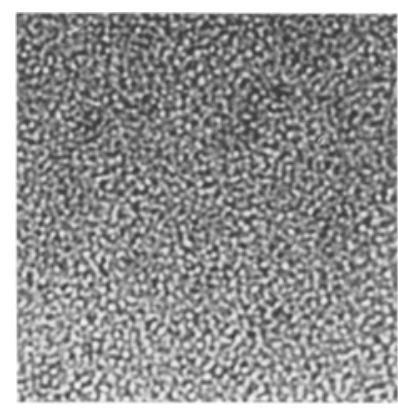

$50^{\circ} \mathrm{C}-15 \mathrm{sec}$

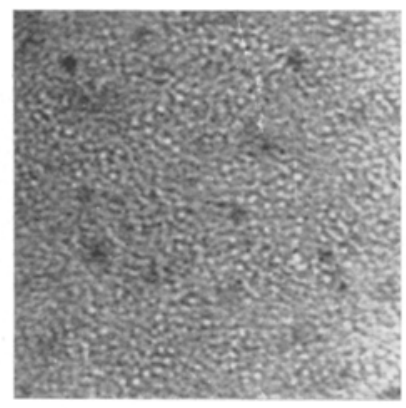

$90{ }^{\circ} \mathrm{C}-10$ sec

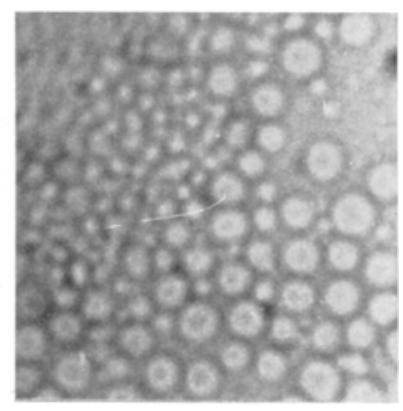

$120^{\circ} \mathrm{C}-1 \mathrm{~min}$

Fig. 10. Initial structures of phase separation of a 15\% Elvax-10/CL solution quenched at different temperatures 


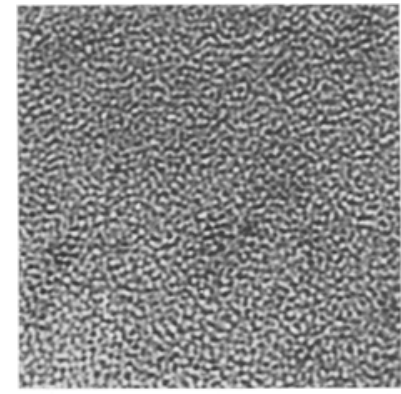

$30 \mathrm{sec}$

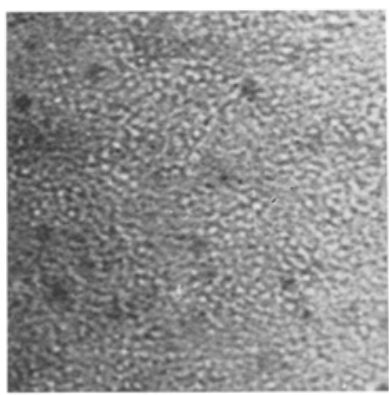

$10 \mathrm{sec}$

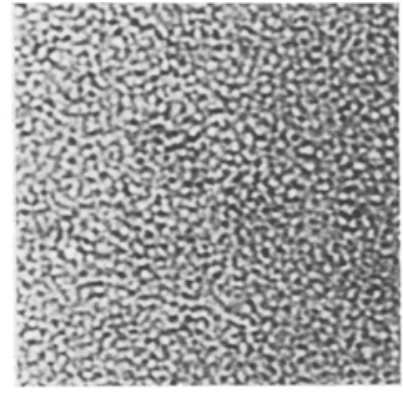

$7 \mathrm{~min}$



$1,5 \mathrm{~min}$

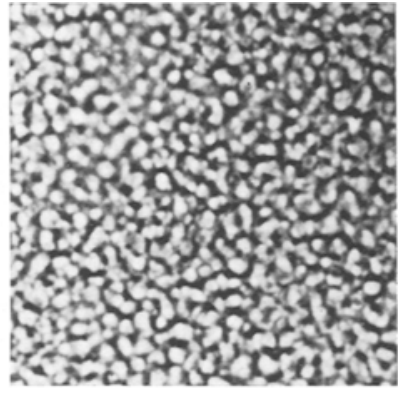

$70 \mathrm{~min}$

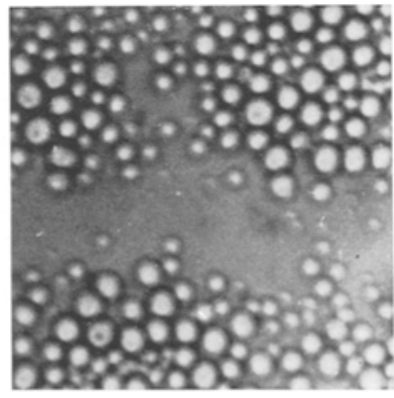

$4 \mathrm{~min}$

Fig. 11. Time study of phase separation in Elvax-40/CL solutions. a) $10 \%$ solution quenched at $50{ }^{\circ} \mathrm{C}$; b) $15 \%$ solution quenched at $90^{\circ} \mathrm{C}$

On observing the phase separation under the microscope one initially sees, at low quenching temperatures, the same phase separation pattern as with PPO/CL. (fig. 10; $50^{\circ} \mathrm{C}$ and $90^{\circ} \mathrm{C}$ ). At higher quenching temperatures drop formation is observed already at relatively short times (fig. $10 ; 120^{\circ} \mathrm{C}$ ). Further changes in these patterns here occur at all the test temperatures (fig. 11, a and b).

\section{Summary}

Two mechanisms of liquid-liquid phase separation in polymer solutions are possible: nucleation and growth of one of the equilibrium phases, and decomposition by a spinodal mechanism. The conditions for and characteristics of both mechanisms are discussed. By combining a number of observations on phase separation in concentrated solutions of poly(2,6-dimethyl-1,4-phenylene ether) and of an ethylene - vinyl acetate copolymer in caprolactam, it becomes clear that a spinodal mechanism is operative at the onset of the separation process. In later stages the flow properties of the concentrated polymer phase determine the final state of the separated mixture.

\section{Zusammenfassung}

Zwei Mechanismen der flüssig-flüssig Phasentrennung in Polymerlösungen sind möglich: 1. Keimbildung und Wachstum einer der Gleichgewichtsphasen und 2. Auftrennung durch einen spinodalen Mechanismus. Die Bedingungen für und das Charakteristische beider Mechanismen wird diskutiert. Durch Kombination einer Zahl von Beobachtungen auf die Phasentrennung in konzentrierten Lösungen aus Poly(2,6-dimethyl-1,4- phenylenäther) und einem Äthylen-vinyl-acetatcopolymer in Caprolactam wird klar, daß ein spinodaler Mechanismus bei Einsatz des Trennprozesses wirksam wird. In späteren Stadien bestimmen die Fließeigenschaften der konzentrierten polymeren Phase den endgültigen $\mathrm{Zu}$ stand der getrennten Mischung.

\section{References}

1) Talen, J.L., G. Challa, Polymer Letters 4, 407 (1966).

2) Pennings, A.J., A.M.Kiel, Kolloid-Z. u. Z. Polymere 205, 160 (1965).

3) Koningsveld, R., A. J. Staverman, Kolloid-Z. u.Z. Polymere 218, 114 (1967); ibid., J. Pol. Sci. Part C 16-3, 1775 (1967); Rehage, G., D. Möller, J. Pol. Sci. Part C 16-3, 1787 (1967).

4) Papkov, S. P., S. G. Yefimova, Kaserf. u. Textiltechn., Sowj. Beitr. 4, 118 (1967).

5) Cahn, J.W.,Acta Met. 9, 795 (1961);Cahn, J.W., J. Chem. Phys. 42, 93 (1965).

6) Hillert, M., Acta Met. 9, 525 (1961); Uhlmann, D. R., B. Chalmers, Ind. Eng. Chem. 57, 19 (1965).

7) See R. J. Charles, Sci. Amer. Sept. 1967, 126

8) Maier, K.H., E. A. Scheurmann, Kolloid-Z. 171, $122(1960)$.

9) van Aartsen, J. J., C. A. Smolders, Europ. Polymer J. 6, $1105(1970)$ $(1970)$

10) van Aartsen, J. J., Europ. Polymer J. 6, 919 Authors' addresses:

Dr. C. A. Smolders

Technological University Twente Enschede (The Netherlands)

Dr. J. J. van Aartsen and A. Steenbergen AKZO, Research Laboratories, Arnhem (The Netherlands) 